\title{
Syntheses, Spectroscopy and X-Ray Crystal Structures of Luminescent Nitrido- and trans-Dioxo-rhenium(v) Complexes of Phosphines and Arsines $\dagger$
}

\author{
Vivian Wing-Wah Yam, ${ }^{*, a}$ Kwok-Kwong Tam, ${ }^{a}$ Ming-Chu Cheng, ${ }^{b}$ Shie-Ming Peng ${ }^{b}$ \\ and Yu Wang ${ }^{b}$ \\ a Department of Chemistry, University of Hong Kong, Pokfulam Road, Hong Kong \\ ${ }^{b}$ Department of Chemistry, National Taiwan University, Taipei, Taiwan
}

\begin{abstract}
Reaction of $\left[\mathrm{ReNCl}_{2}\left(\mathrm{PPh}_{3}\right)_{2}\right]$ and $\left[\mathrm{ReO}_{2}\left(\mathrm{PPh}_{3}\right)_{2} 1\right]$ with a variety of phosphines and arsines, $\mathrm{L}$, yielded the respective complex cations $\left[\mathrm{ReNL}_{2} \mathrm{Cl}^{+}{ }^{+}\right.$and trans- $\left[\mathrm{ReO}_{2} \mathrm{~L}_{2}\right]^{+}$. The $\mathrm{X}$-ray crystal structures of $\left[\mathrm{ReN}(\mathrm{dpae})_{2} \mathrm{Cl}\right]^{+}$and trans- $\left[\mathrm{ReO}_{2}(\mathrm{dadpe})_{2}\right]^{+}$[dpae $=1,2$-bis(diphenylarsino)ethane, dadpe $=1$ diphenylarsino-2-diphenylphosphinoethane] have been determined: $\left[\mathrm{ReN}(\mathrm{dpae})_{2} \mathrm{Cl}\right]^{+}$, monoclinic, space group $C c, a=10.362(2), \quad b=22.823(5), \quad c=21.363(6) \quad \AA, \quad \beta=92.74(2)^{\circ}, Z=4$; trans$\left[\mathrm{ReO}_{2}(\text { dadpe })_{2}\right]^{+}$, monoclinic, space group $C c, a=9.868(3), b=22.608(5), c=21.887(8) \AA, \beta=$ $91.87(3)^{\circ}, Z=4$. A $R e=N$ distance of $1.839(8)$ and $R e=0$ distances of $1.785(8)$ and $1.766(8) \AA$ have been measured. The $\left[\mathrm{ReNL} \mathrm{Cl}_{2}\right]^{+}$and trans $-\left[\mathrm{ReO}_{2} \mathrm{~L}_{2}\right]^{+}$complexes exhibit intense $v(\mathrm{Re}=\mathrm{N})$ and $v_{\text {asym }}\left(\mathrm{ReO}_{2}\right)$ stretches at $1043-1049$ and $785-790 \mathrm{~cm}^{-1}$, respectively. Excitation of solid samples of $\left[\mathrm{ReNL}{ }_{2} \mathrm{Cl}_{1} \mathrm{ClO}_{4}\right.$ and trans- $\left[\mathrm{ReO}_{2} \mathrm{~L}_{2}\right] \mathrm{ClO}_{4}$ at $350-380 \mathrm{~nm}$ at room temperature results in yellow-green and orange emission, respectively. Both $\left[\operatorname{ReN}(\mathrm{dppbz})_{2} \mathrm{Cl}\right]^{+}$and $\left[\operatorname{ReN}(R-1,2-\mathrm{dppp})_{2} \mathrm{Cl}\right]^{+}[\mathrm{dppbz}=$ 1,2-bis(diphenylphosphine)benzene, $\quad R$-1,2-dppp $=R-1,2$-bis(diphenylphosphino)propane] exhibit intense long-lived room-temperature luminescence in fluid solutions. Concentration quenching has been observed for both $\left[\operatorname{ReN}(\mathrm{dppbz})_{2} \mathrm{Cl}\right]^{+}$and $\left[\operatorname{ReN}(R-1,2-\mathrm{dppp})_{2} \mathrm{Cl}\right]^{+}$in $\mathrm{MeCN}\left(0.1 \mathrm{~mol} \mathrm{dm}^{-3}\right.$ $\mathrm{NBu}_{4} \mathrm{PF}_{6}$ ) with self-quenching rate constants of ca. $5.7 \times 10^{7}$ and $3.2 \times 10^{8} \mathrm{dm}^{3} \mathrm{~mol}^{-1} \mathrm{~s}^{-1}$, respectively, and inherent lifetimes of ca. 15 and $0.9 \mu \mathrm{s}$ at infinite dilution. All the trans- $\left[\mathrm{ReO}_{2} \mathrm{~L}_{2}\right]^{+}$ complexes exhibit room-temperature luminescence in $\mathrm{MeCN}$. Cyclic voltammetric studies show that the $\left[\mathrm{ReNL}_{2} \mathrm{Cl}^{+}\right.$complexes display an irreversible reduction couple at $E_{\mathrm{pc}}$ of ca. -2.1 to -2.4 and an irreversible oxidation couple at $E_{\mathrm{pa}}$ of $c a$. +1.4 to $+1.7 \mathrm{~V}$ vs. ferrocene-ferrocenium in $\mathrm{MeCN}(0.1 \mathrm{~mol}$ $\mathrm{dm}^{-3} \mathrm{NBu}_{4} \mathrm{PF}_{6}$ ). Irreversible reduction and oxidation couples are also observed for the trans$\left[\mathrm{ReO}_{2} \mathrm{~L}_{2}\right]^{+}$species. A direct comparison of the photophysical and spectroscopic properties of $\left[\mathrm{ReNL} \mathrm{Cl}_{2}\right]^{+}$and trans $-\left[\mathrm{ReO}_{2} \mathrm{~L}_{2}\right]^{+}$has been made.
\end{abstract}

Recently, there has been considerable interest in the spectroscopic as well as photophysical studies of luminescent metaloxo complexes, ${ }^{1}$ of which the trans-dioxorhenium(v) and osmium(vI) complexes of nitrogen-donor ligands are the most well studied. Studies on the isoelectronic nitrido complexes are less extensive and have been mainly confined to those of osmium(vI). ${ }^{2}$ In this paper, we report on the syntheses, characterization and photophysical properties of a series of new nitrido- and trans-dioxo-rhenium(v) complexes of phosphine and arsine ligands, which provide a systematic direct comparison of the isoelectronic oxo and nitrido moieties with the same set of spectator ligands. The X-ray crystal structures of a nitrido and a trans-dioxo complex of rhenium( $(\mathrm{v})$ are described.

\section{Experimental}

Reagents and Materials.-Both rhenium powder and potassium perrhenate(vII) were purchased from Johnson Matthey Ltd. The complexes $\left[\mathrm{ReNCl}_{2}\left(\mathrm{PPh}_{3}\right)_{2}\right],{ }^{3 a}\left[\mathrm{ReN}(\mathrm{dppe})_{2} \mathrm{Cl}\right] \mathrm{Cl}^{3 b}$ and $\left[\mathrm{ReO}_{2}\left(\mathrm{PPh}_{3}\right)_{2} \mathrm{I}\right]^{3 \mathrm{c}}$ were prepared according to literature procedures except that $\mathrm{KReO}_{4}$ was used in place of perrhenic acid in the preparation of $\left[\mathrm{ReO}_{2}\left(\mathrm{PPh}_{3}\right)_{2} \mathrm{I}\right]$. The ligands

† Supplementary data available: see Instructions for Authors, J. Chem. Soc., Dalton Trans., 1992, Issue 1, pp. xx-xxv.

Non-SI unit employed: $\mathrm{eV} \approx 1.6 \times 10^{-19} \mathrm{~J}$ 1,2-bis(diphenylphosphino)ethane (dppe), 1,2-bis(diphenylphosphino)benzene (dppbz), $R$ - $(+)$-1,2-bis(diphenylphosphino)propane ( $R$-1,2-dppp) and 1-diphenylarsino-2-diphenylphosphinoethane (dadpe) were obtained from Strem Chemicals, Inc.; 1,2-bis(diphenylarsino)ethane (dpae) was obtained from Aldrich Chemical Co. Tetra- $n$-butylammonium hexafluorophosphate (Aldrich) was recrystallized twice from absolute ethanol and vacuum dried before use. Both dichloromethane (AJAX, AR) and acetonitrile (Mallinckrodt, ChromAR) were distilled over calcium hydride before use. All other reagents and solvents were of analytical grade and were used as received.

Physical Measurements and Instrumentation.--The UV/VIS spectra were obtained on a Milton Roy Spectronic 3000 diode array spectrophotometer, IR spectra as Nujol mulls on a Shimadzu IR-470 infrared spectrophotometer $\left(4000-400 \mathrm{~cm}^{-1}\right)$, steady-state emission spectra on a Hitachi 650-60 fluorescence spectrophotometer. Phosphorus-31 NMR spectra were recorded on a JEOL JNM-GSX270 Fourier-transform NMR spectrometer with chemical shifts reported relative to $\mathrm{H}_{3} \mathrm{PO}_{4}$. Conductivity measurements were made using a Radiometer model $\mathrm{CDH} 2$ conductivity meter with $0.1 \mathrm{~mol} \mathrm{dm}^{-3} \mathrm{KCl}$ as calibrant. Magnetic susceptibility measurements were made using the Gouy method. Elemental analyses of the new complexes were performed either by the Butterworth Laboratories or the Shanghai Institute of Organic Chemistry, Academia Sinica. 
Emission-lifetime measurements were performed using a conventional laser system. The excitation source was the 355nm output (third harmonic) of a Quanta-Ray Q-switched DCR3 pulsed Nd-YAG laser $(10 \mathrm{~Hz}, \mathrm{G}$-resonator). Luminescence decay signals were recorded on a Tektronix model 2430 digital oscilloscope, and analysed using a program for exponential fits. All solutions for photophysical studies were prepared under vacuum in a $10 \mathrm{~cm}^{3}$ round bottom flask equipped with a sidearm $1 \mathrm{~cm}$ fluorescence cuvette and sealed from the atmosphere by a Kontes quick-release Teflon stopper. Solutions were rigorously degassed with no fewer than four freeze-pumpthaw cycles. Concentration quenching experiments were monitored by time-resolved (lifetime) emission measurements in $\mathrm{MeCN}\left(0.1 \mathrm{~mol} \mathrm{dm}{ }^{-3} \mathrm{NBu}_{4} \mathrm{PF}_{6}\right)$ and data were treated by a Stern-Volmer fit as described by $\tau_{0} / \tau=1+k_{\mathrm{q}} \tau_{0}[\mathrm{Re}]$, where $\tau_{0}$ and $\tau$ are the respective excited-state lifetimes at infinite dilution and at various concentrations of the metal complex [Re], and $k_{\mathrm{q}}$ is the bimolecular self-quenching rate constant.

Cyclic voltammetric measurements were performed by using a Princeton Applied Research (PAR) universal programmer (model 175), potentiostat (model 173), and digital coulometer (model 179). The ferrocenium-ferrocene couple was used as the internal standard ${ }^{4}$ in the electrochemical measurements in acetonitrile $\left(0.1 \mathrm{~mol} \mathrm{dm}^{-3} \mathrm{NBu}_{4} \mathrm{PF}_{6}\right)$. The working electrode was a glassy carbon (Atomergic Chemicals V25) electrode with a platinum foil acting as the counter electrode. Treatment of the electrode surfaces was as reported elsewhere. ${ }^{5}$

Syntheses of Rhenium Complexes.- $\left[\mathrm{ReNL}_{2} \mathrm{Cl}\right] \mathrm{Y}(\mathrm{L}=$ dppe, $d p p b=$, dpae, dadpe or $\mathrm{R}-1,2-d p p p ; \mathrm{Y}=\mathrm{Cl}$ or $\left.\mathrm{ClO}_{4}\right)$. These complexes were prepared by a method similar to that reported by Johnson. ${ }^{3 b}$ A suspension of $\left[\mathrm{ReNCl}_{2}\left(\mathrm{PPh}_{3}\right)_{2}\right](0.2 \mathrm{~g}, 0.25 \mathrm{mmol})$ and $\mathrm{L}(0.55 \mathrm{mmol})$ in benzene $\left(10 \mathrm{~cm}^{3}\right)$ was refluxed under an atmosphere of nitrogen for $c a .4 \mathrm{~h}$ during which the brick-red suspension turned yellow. The reaction mixture was then rotary evaporated to dryness. The yellow solid was then recrystallized from methanol-diethyl ether to give crystals of $\left[\mathrm{ReNL}_{2} \mathrm{Cl}\right] \mathrm{Cl}$. The perchlorate salts were obtained by metathesis of the chloride salts in methanol using lithium perchlorate. Crystalline samples of $\left[\mathrm{ReNL}_{2} \mathrm{Cl}\right] \mathrm{ClO}_{4}$ were obtained by recrystallization via diffusion of diethyl ether into an acetonitrile solution of the complex. An alternative procedure using methanol in place of benzene for the reaction of $\left[\mathrm{ReNCl}_{2}\left(\mathrm{PPh}_{3}\right)_{2}\right]$ and $\mathrm{L}$ followed by addition of $\mathrm{LiClO}_{4}$ also afforded $\left[\mathrm{ReNL}_{2} \mathrm{Cl}_{3} \mathrm{ClO}_{4}\right.$

$\left[\mathrm{ReN}(\mathrm{dppe}){ }_{2} \mathrm{Cl}\right] \mathrm{ClO}_{4}$ (Found: $\mathrm{C}, 55.0 ; \mathrm{H}, 4.2 ; \mathrm{Cl}, 6.3 ; \mathrm{N}, 1.1$. Calc.: C, 55.2; H, 4.3; Cl, 6.3; N, 1.2\%); IR (Nujol mull), v(Re=N) $1043 \mathrm{~cm}^{-1}$; magnetic moment (at $\left.298 \mathrm{~K}\right), \mu_{\text {eff }} 0$; UV/VIS (MeCN), $\lambda / \mathrm{nm}\left(\varepsilon_{\max } / \mathrm{dm}^{3} \mathrm{~mol}^{-1} \mathrm{~cm}^{-1}\right) 221$ (71 160), 239 (sh) (59 710), 269 (sh) (19 080), 307 (sh) (990) and 369 (250); ${ }^{31} \mathrm{P}$ NMR (MeCN), $\delta 27.89$; molar conductivity $(298 \mathrm{~K}$ ) (in MeCN) 132 (in $\mathrm{MeOH}$ ) $87 \Omega^{-1} \mathrm{~cm}^{2} \mathrm{~mol}^{-1}$. [ReN(dppbz) $\left.{ }_{2} \mathrm{Cl}\right] \mathrm{ClO}_{4}{ }^{*}$ MeCN (Found: C, 58.6; H, 4.0; N, 2.0. Calc.: C, 58.7; H, 4.1; N, $2.2 \%$ ); IR (Nujol mull), $v(\operatorname{Re} \equiv \mathrm{N}) 1043 \mathrm{~cm}^{-1}$; magnetic moment (at $298 \mathrm{~K}), \mu_{\text {eff }} 0$; UV/VIS $(\mathrm{MeCN}), \lambda / \mathrm{nm}\left(\varepsilon_{\max } / \mathrm{dm}^{3} \mathrm{~mol}^{-1}\right.$ $\mathrm{cm}^{-1}$ ) 221 (100 410), 244 (sh) (56 285), 269 (sh) (21 130), 278 (sh) (17 250), 287 (sh) (9640), 307 (sh) (1320), 360 (330), 427 (sh) (90) and 472 (sh) (60); ${ }^{31} \mathrm{P}$ NMR (MeCN), $833.38,(\mathrm{MeCN}-\mathrm{MeOH}$ $1: 1 \mathrm{v} / \mathrm{v}) \delta 33.23$; molar conductivity $(298 \mathrm{~K}$ ) (in MeCN) 134 (in $\mathrm{MeOH}) 89 \Omega^{-1} \mathrm{~cm}^{2} \mathrm{~mol}^{-1}$. [ReN(dpae) $\left.{ }_{2} \mathrm{Cl}\right] \mathrm{ClO}_{4}$ (Found: C, 47.8; H, 3.5; N, 1.0. Calc.: C, 47.8; H, 3.7; N, 1.1\%); IR (Nujol mull), $v(\mathrm{Re} \equiv \mathrm{N}) 1044 \mathrm{~cm}^{-1}$; magnetic moment (at $\left.298 \mathrm{~K}\right), \mu_{\mathrm{eff}} 0$; UV/VIS (MeCN), $\lambda / \mathrm{nm}\left(\varepsilon_{\max } / \mathrm{dm}^{3} \mathrm{~mol}^{-1} \mathrm{~cm}^{-1}\right) 219$ (74 850), 238 (sh) (59 610), 266 (sh) (18 490), 307 (sh) (750) and 372 (280). For $\left[\mathrm{ReN}(\text { dadpe })_{2} \mathrm{Cl}\right] \mathrm{ClO}_{4}$, both yellow and orange forms occur. Attempts to isolate pure forms of each isomer are difficult due to the relative ease of isomerization (Found: $\mathrm{C}, 50.9 ; \mathrm{H}, 3.8$; $\mathrm{Cl}, 5.8 ; \mathrm{N}, 1.0$. Calc.: C, 51.2; H, 4.0; Cl, 5.8; N, 1.2\%); IR (Nujol mull), $v(\mathrm{Re} \equiv \mathrm{N}) 1043 \mathrm{~cm}^{-1}$; magnetic moment (at $298 \mathrm{~K}$ ), $\mu_{\mathrm{eff}} 0$; UV/VIS (MeCN), $\lambda / \mathrm{nm}\left(\varepsilon_{\max } / \mathrm{dm}^{3} \mathrm{~mol}^{-1} \mathrm{~cm}^{-1}\right) 204(116400)$, 238 (sh) (57 010), 266 (sh) (19 800), 307 (sh) (900) and 372 (245); ${ }^{31} \mathrm{P}$ NMR $(\mathrm{MeCN}), \delta 35.18,36.84$. $\left[\mathrm{ReN}(R-1,2 \text {-dppp })_{2} \mathrm{Cl} \mathrm{ClO}_{4}\right.$
(Found: C, 55.7; H, 4.5; N, 1.1. Calc.: C, 55.9; H, 4.5; N, 1.2\%); IR (Nujol mull), $v(\mathrm{Re} \equiv \mathrm{N}) 1049 \mathrm{~cm}^{-1}$; magnetic moment (at $298 \mathrm{~K}$ ), $\mu_{\mathrm{eff}} 0$; UV/VIS (MeCN), $\lambda / \mathrm{nm}\left(\varepsilon_{\max } / \mathrm{dm}^{3} \mathrm{~mol}^{-1} \mathrm{~cm}^{-1}\right) 219$ (70 890), 242 (sh) (56 420), 270 (sh) (20 850), 307 (sh) (945) and 374 (250); ${ }^{31}$ P NMR (MeCN), $\delta 12.01$ and 35.34 .

$\left[\mathrm{ReO}_{2} \mathrm{~L}_{2}\right] \mathrm{Y}(\mathrm{L}=d p p e, d p p b z$, dpae, dadpe or $\mathrm{R}-1,2-d p p p$; $\mathrm{Y}=\mathrm{I}$ or $\mathrm{ClO}_{4}$ ). These complexes were prepared by a method similar to that reported by Brewer and Gray. ${ }^{6}$ A suspension of $\left[\mathrm{ReO}_{2}\left(\mathrm{PPh}_{3}\right)_{2} \mathrm{I}\right](0.2 \mathrm{~g}, 0.23 \mathrm{mmol})$ and $\mathrm{L}(0.51 \mathrm{mmol})$ in methanol $\left(10 \mathrm{~cm}^{3}\right)$ was refluxed for $c a .4 \mathrm{~h}$ during which the violet suspension turned to a clear yellow solution. The reaction mixture was then reduced in volume to afford yellow crystals of $\left[\mathrm{ReO}_{2} \mathrm{~L}_{2}\right] \mathrm{I}$, which were recrystallized from methanol-diethyl ether. The perchlorate salts were obtained by metathesis of the iodide salts in methanol using lithium perchlorate. Crystalline samples of $\left[\mathrm{ReO}_{2} \mathrm{~L}_{2}\right] \mathrm{ClO}_{4}$ were obtained by recrystallization via diffusion of diethyl ether into an acetonitrile solution of the complex.

$\left[\mathrm{ReO}_{2}(\text { dppe })_{2}\right] \mathrm{ClO}_{4} \cdot \mathrm{H}_{2} \mathrm{O}$ (Found: C, 55.3; $\mathrm{H}, 4.2 ; \mathrm{Cl}, 2.9$. Calc.: C, $55.2 ; \mathrm{H}, 4.5 ; \mathrm{Cl}, 3.1 \%$ ); IR (Nujol mull), $v_{\text {asym }}(\mathrm{Re}=\mathrm{O}) 784$ $\mathrm{cm}^{-1}$; magnetic moment (at $298 \mathrm{~K}$ ), $\mu_{\text {eff }} 0$; UV/VIS (MeCN), $\lambda / \mathrm{nm}\left(\varepsilon_{\max } / \mathrm{dm}^{3} \mathrm{~mol}^{-1} \mathrm{~cm}^{-1}\right) 268$ (42 615), 309 (sh) (2520) and 389 (sh) (207). [ $\left.\mathrm{ReO}_{2}(\mathrm{dppbz})_{2}\right] \mathrm{ClO}_{4} \cdot \mathrm{MeCN} \cdot \mathrm{H}_{2} \mathrm{O}$ (Found: $\mathrm{C}$, 58.6; H, 4.0; Cl, 2.6; N, 0.9. Calc.: C, 58.7; H, 4.2; Cl, 2.8; N, $1.1 \%$ ); IR (Nujol mull), $v_{\text {asym }}(\mathrm{Re}=\mathrm{O}) 790 \mathrm{~cm}^{-1}$; magnetic moment (at $298 \mathrm{~K}), \mu_{\mathrm{eff}} 0 ; \mathrm{UV} / \mathrm{VIS}(\mathrm{MeCN}), \lambda / \mathrm{nm}\left(\varepsilon_{\max } / \mathrm{dm}^{3} \mathrm{~mol}^{-1} \mathrm{~cm}^{-1}\right) 240$ (sh) (50 860), 270 (sh) (28 590), 290 (sh) (18 715) and 389 (sh) (235); ${ }^{31} \mathrm{P}$ NMR (MeCN), $\delta$ 15.45; molar conductivity (at $298 \mathrm{~K}$ ) (in $\mathrm{MeCN}$ ) 129 , (in $\mathrm{MeOH}$ ) $83 \Omega^{-1} \mathrm{~cm}^{2} \mathrm{~mol}^{-1}$ $\left[\mathrm{ReO}_{2}\right.$ (dpae) $\left.{ }_{2}\right] \mathrm{ClO}_{4} \cdot \mathrm{H}_{2} \mathrm{O}$ (Found: $\mathrm{C}, 47.9 ; \mathrm{H}, 3.5 ; \mathrm{Cl}$, 2.6. Calc.: $\mathrm{C}, 47.7 ; \mathrm{H}, 3.8 ; \mathrm{Cl}, 2.7 \%$ ); IR (Nujol mull), $v_{\text {asym }}(\mathrm{Re}=\mathrm{O}) 786 \mathrm{~cm}^{-1}$; magnetic moment (at $298 \mathrm{~K}$ ), $\mu_{\text {eff }} 0$; UV/VIS (MeCN), $\lambda / \mathrm{nm}$ $\left(\varepsilon_{\max } / \mathrm{dm}^{3} \mathrm{~mol}^{-1} \mathrm{~cm}^{-1}\right) 243(\mathrm{sh})(41040), 273$ (52 890), $312(\mathrm{sh})$ (2950) and 389 (sh) (295). [ $\left.\mathrm{ReO}_{2}(\text { dadpe })_{2}\right] \mathrm{ClO}_{4} \cdot \mathrm{H}_{2} \mathrm{O}$ (Found: C, 51.4; H, 3.8; Cl, 2.7. Calc.: C, 51.2; H, 4.1; Cl, $2.9 \%$ ); IR (Nujol mull), $v_{\text {asym }}(\mathrm{Re}=\mathrm{O}) 786 \mathrm{~cm}^{-1}$; magnetic moment (at $\left.298 \mathrm{~K}\right), \mu_{\text {eff }}$ 0 ; UV/VIS $(\mathrm{MeCN}), \lambda / \mathrm{nm}\left(\varepsilon_{\max } / \mathrm{dm}^{3} \mathrm{~mol}^{-1} \mathrm{~cm}^{-1}\right) 244(\mathrm{sh})$ (41 310), 270 (56 070), 312 (sh) (2670) and 395 (sh) (220); ${ }^{31} \mathrm{P}$ NMR (MeCN), $\delta 15.48$ and 19.09. $\left[\mathrm{ReO}_{2}(R-1,2-\mathrm{dppp})_{2}\right] \mathrm{ClO}_{4}$. $2 \mathrm{H}_{2} \mathrm{O}$ (Found: $\mathrm{C}, 55.2 ; \mathrm{H}, 4.2 ; \mathrm{Cl}, 2.7$. Calc.: C, 55.0; $\mathrm{H}, 4.8 ; \mathrm{Cl}$, $3.0 \%$ ); IR (Nujol mull), $v_{\text {asym }}(\mathrm{Re}=\mathrm{O}) 786 \mathrm{~cm}^{-1}$; magnetic moment (at $298 \mathrm{~K}$ ), $\mu_{\text {eff }} 0$; UV/VIS (MeCN), $\lambda / \mathrm{nm}\left(\varepsilon_{\max } / \mathrm{dm}^{3}\right.$ $\left.\mathrm{mol}^{-1} \mathrm{~cm}^{-1}\right) 273(35620), 312(\mathrm{sh})(2540)$ and $400(\mathrm{sh})(175)$.

Crystal Structure Determination.- $\left[\mathrm{ReN}(\mathrm{dpae})_{2} \mathrm{Cl}_{3} \mathrm{ClO}_{4}\right.$. The complex $\left[\mathrm{ReN}(\mathrm{dpae})_{2} \mathrm{Cl}_{3} \mathrm{ClO}_{4}\right.$ was recrystallized by vapour diffusion of diethyl ether into acetonitrile.

Crystal data. $\mathrm{C}_{52} \mathrm{H}_{48} \mathrm{As}_{4} \mathrm{Cl}_{2} \mathrm{NO}_{4} \mathrm{Re}, M_{\mathrm{r}}=1307.8$, monoclinic, space group $C c, a=10.362(2), b=22.823(5), c=$ $21.363(6) \AA, \beta=92.74(2)^{\circ}, U=5046(2) \AA^{3}, Z=4, D_{\mathrm{c}}=$ $1.722 \mathrm{~g} \mathrm{~cm} \mathrm{~cm}^{-3}, \quad F(000)=2560, \quad$ crystal dimensions $=$ $0.30 \times 0.50 \times 0.50 \mathrm{~mm}$. The intensity data were measured on a CAD-4 diffractometer using Mo-K $\alpha$ radiation $(\lambda=0.7107 \AA)$ using the $\omega-2 \theta$ scan mode at $297 \mathrm{~K}$ with $2 \theta_{\max }=50^{\circ}$. Cell dimensions were obtained from 24 reflections with $2 \theta$ angles in the range $19.12-21.58^{\circ} .4698$ Reflections were measured of which 3793 were observed $[I>2.0 \sigma(I)]$. Absorption corrections $\left(\mu=38.8 \mathrm{~cm}^{-1}\right)$ were made according to $\psi$ curves of three selected reflections. The minimum and maximum transmission factors are 0.77 and 1.00 . The structure was solved by the Patterson method and refined by least squares. Full-matrix least-squares refinement on 576 parameters converged to yield agreement indices $R=0.033, R^{\prime}=0.020$ and goodness of fit $=$ 1.83; $\max . \Delta / \sigma=0.19$; the residual $\Delta \rho< \pm 0.97 \mathrm{e} \AA^{-3}$; weights are based on counting statistics. Table 1 lists the atomic coordinates of non-hydrogen atoms. Selected bond distances and angles are summarized in Table 2 . All the hydrogen atom parameters were calculated from the idealized geometry.

$\left[\mathrm{ReO}_{2}(\text { dadpe })_{2}\right] \mathrm{ClO}_{4}$. The complex $\left[\mathrm{ReO}_{2}(\text { dadpe })_{2}\right] \mathrm{ClO}_{4}$ was recrystallized by vapour diffusion of diethyl ether into acetonitrile. 
Table 1 Atomic parameters for $\left[\mathrm{ReN}(\mathrm{dpae}){ }_{2} \mathrm{Cl}\right] \mathrm{ClO}_{4}$ with estimated standard deviations (e.s.d.s) in parentheses

\begin{tabular}{|c|c|c|c|c|c|c|c|}
\hline Atom & $x$ & $y$ & $z$ & Atom & $x$ & $y$ & $z$ \\
\hline $\operatorname{Re}$ & 0.89977 & $0.80173(2)$ & 0.84639 & $C(24 B)$ & $0.4218(12)$ & $0.9296(6)$ & $0.9482(6)$ \\
\hline As(1) & $0.9255(1)$ & $0.7030(1)$ & $0.8996(1)$ & $C(25 B)$ & $0.4284(11)$ & $0.8770(6)$ & $0.9149(7)$ \\
\hline As(2) & $0.8119(1)$ & $0.8276(1)$ & $0.9525(1)$ & $C(26 B)$ & $0.5466(10)$ & $0.8489(5)$ & $0.9179(6)$ \\
\hline As(3) & $0.9088(1)$ & $0.9029(1)$ & $0.7971(1)$ & $C(31 A)$ & $0.7697(9)$ & $0.9296(4)$ & $0.7429(4)$ \\
\hline As(4) & $1.0128(1)$ & $0.7751(1)$ & $0.7471(1)$ & $C(32 A)$ & $0.7817(11)$ & $0.9783(5)$ & $0.7058(5)$ \\
\hline $\mathrm{Cl}(5)$ & $1.1148(3)$ & $0.8344(1)$ & $0.8845(1)$ & $C(33 A)$ & $0.6808(12)$ & $0.9987(5)$ & $0.6675(6)$ \\
\hline$N(6)$ & $0.7377(7)$ & $0.7814(3)$ & $0.8148(3)$ & $C(34 A)$ & $0.5647(10)$ & $0.9693(5)$ & $0.6640(5)$ \\
\hline$C(1)$ & $0.7772(9)$ & $0.7005(4)$ & $0.9538(5)$ & $C(35 A)$ & $0.5452(9)$ & $0.9227(4)$ & $0.6987(6)$ \\
\hline$C(2)$ & $0.7723(11)$ & $0.7526(4)$ & $0.9952(5)$ & $C(36 A)$ & $0.6501(10)$ & $0.9036(4)$ & $0.7395(5)$ \\
\hline$C(3)$ & $1.0533(9)$ & $0.9010(4)$ & $0.7410(5)$ & $C(31 B)$ & $0.9426(9)$ & $0.9711(4)$ & $0.8477(5)$ \\
\hline$C(4)$ & $1.0419(12)$ & $0.8466(5)$ & $0.7003(5)$ & $C(32 B)$ & $1.0668(9)$ & $0.9922(4)$ & $0.8602(5)$ \\
\hline$C(11 A)$ & $1.0771(8)$ & $0.6854(4)$ & $0.9530(4)$ & $C(33 B)$ & $1.0880(10)$ & $1.0386(4)$ & $0.9004(5)$ \\
\hline$C(12 A)$ & $1.0669(10)$ & $0.6540(5)$ & $1.0090(5)$ & $C(34 B)$ & $0.9861(11)$ & $1.0642(4)$ & $0.9284(5)$ \\
\hline$C(13 A)$ & $1.1756(11)$ & $0.6412(6)$ & $1.0448(5)$ & $C(35 B)$ & $0.8611(11)$ & $1.0444(5)$ & $0.9145(5)$ \\
\hline$C(14 A)$ & $1.2934(11)$ & $0.6593(5)$ & $1.0296(5)$ & $C(36 B)$ & $0.8409(9)$ & $0.9969(4)$ & $0.8750(5)$ \\
\hline$C(15 A)$ & $1.3045(10)$ & $0.6925(5)$ & $0.9754(5)$ & $C(41 A)$ & $0.9219(11)$ & $0.7324(4)$ & $0.6817(5)$ \\
\hline$C(16 A)$ & $1.1969(9)$ & $0.7052(4)$ & $0.9379(4)$ & $C(42 A)$ & $0.7919(10)$ & $0.7373(5)$ & $0.6713(5)$ \\
\hline$C(11 B)$ & $0.8943(10)$ & $0.6341(3)$ & $0.8481(5)$ & $C(43 A)$ & $0.7277(11)$ & $0.7109(5)$ & $0.6201(6)$ \\
\hline$C(12 B)$ & $0.8099(11)$ & $0.6362(4)$ & $0.8000(5)$ & $C(44 A)$ & $0.7988(14)$ & $0.6771(6)$ & $0.5829(6)$ \\
\hline$C(13 B)$ & $0.7870(12)$ & $0.5889(5)$ & $0.7593(6)$ & $C(45 A)$ & $0.9246(14)$ & $0.6659(7)$ & $0.5921(7)$ \\
\hline$C(14 B)$ & $0.8539(12)$ & $0.5386(5)$ & $0.7689(6)$ & $C(46 A)$ & $0.9842(11)$ & $0.6973(6)$ & $0.6419(6)$ \\
\hline$C(15 B)$ & $0.9398(12)$ & $0.5363(4)$ & $0.8174(6)$ & $C(41 B)$ & $1.1768(9)$ & $0.7363(5)$ & $0.7587(5)$ \\
\hline$C(16 B)$ & $0.9674(9)$ & $0.5824(4)$ & $0.8583(5)$ & $C(42 B)$ & $1.1840(11)$ & $0.6762(5)$ & $0.7705(6)$ \\
\hline$C(21 A)$ & $0.9077(9)$ & $0.8680(4)$ & $1.0182(4)$ & $C(43 B)$ & $1.3005(15)$ & $0.6502(6)$ & $0.7833(7)$ \\
\hline$C(22 A)$ & $0.8860(10)$ & $0.8573(5)$ & $1.0803(5)$ & $C(44 B)$ & $1.4032(12)$ & $0.6852(6)$ & $0.7841(6)$ \\
\hline$C(23 A)$ & $0.9509(11)$ & $0.8906(5)$ & $1.1264(5)$ & $C(45 B)$ & $1.4102(13)$ & $0.7399(7)$ & $0.7695(8)$ \\
\hline$C(24 A)$ & $1.0327(11)$ & $0.9340(5)$ & $1.1115(5)$ & $C(46 B)$ & $1.2874(12)$ & $0.7665(6)$ & $0.7597(8)$ \\
\hline$C(25 A)$ & $1.0573(12)$ & $0.9449(5)$ & $1.0500(5)$ & $\mathrm{Cl}$ & $0.2423(3)$ & $0.9127(1)$ & $0.5859(1)$ \\
\hline$C(26 A)$ & $0.9954(10)$ & $0.9111(4)$ & $1.0042(5)$ & $\mathrm{O}(1)$ & $0.3068(7)$ & $0.8610(3)$ & $0.6056(3)$ \\
\hline$C(21 B)$ & $0.6507(9)$ & $0.8715(4)$ & $0.9511(5)$ & $\mathbf{O}(2)$ & $0.2410(7)$ & $0.9522(3)$ & $0.6360(4)$ \\
\hline$C(22 B)$ & $0.6371(11)$ & $0.9236(5)$ & $0.9814(5)$ & $\mathrm{O}(3)$ & $0.1149(7)$ & $0.8996(4)$ & $0.5660(4)$ \\
\hline$C(23 B)$ & $0.5245(12)$ & $0.9540(5)$ & $0.9800(6)$ & $\mathrm{O}(4)$ & $0.3049(7)$ & $0.9384(3)$ & $0.5355(3)$ \\
\hline
\end{tabular}

Table 2 Selected bond distances $(\AA)$ and angles $\left(^{\circ}\right)$ for $[\operatorname{ReN}$ $\left.(\mathrm{dpae})_{2} \mathrm{Cl}\right]^{+}$with e.s.d.s in parentheses

\begin{tabular}{lllr}
$\operatorname{Re}-\mathrm{N}(6)$ & $1.839(8)$ & $\operatorname{Re}-\mathrm{Cl}(5)$ & $2.451(3)$ \\
$\operatorname{Re}-\mathrm{As}(1)$ & $2.532(1)$ & $\operatorname{Re}-\mathrm{As}(2)$ & $2.552(1)$ \\
$\operatorname{Re}-\mathrm{As}(3)$ & $2.541(1)$ & $\operatorname{Re}-\mathrm{As}(4)$ & $2.546(1)$ \\
$\mathrm{As}(1)-\mathrm{C}(1)$ & $1.968(10)$ & $\mathrm{As}(2)-\mathrm{C}(2)$ & $1.991(10)$ \\
$\mathrm{As}(3)-\mathrm{C}(3)$ & $1.962(9)$ & $\mathrm{As}(4)-\mathrm{C}(4)$ & $1.945(10)$ \\
$\mathrm{As}-\mathrm{C}(\mathrm{Ph})$ & $1.905(10)-1.946(10)$ & & \\
$\mathrm{As}(1)-\operatorname{Re}-\mathrm{As}(2)$ & $80.86(4)$ & $\mathrm{As}(3)-\operatorname{Re}-\mathrm{As}(4)$ & $81.04(4)$ \\
$\mathrm{As}(1)-\operatorname{Re}-\mathrm{As}(3)$ & $171.65(3)$ & $\mathrm{As}(2)-\operatorname{Re}-\mathrm{As}(4)$ & $173.42(4)$ \\
$\mathrm{As}(1)-\operatorname{Re}-\mathrm{As}(4)$ & $96.82(4)$ & $\mathrm{As}(2)-\operatorname{Re}-\mathrm{As}(3)$ & $100.37(4)$ \\
$\mathrm{Cl}(5)-\operatorname{Re}-\mathrm{N}(6)$ & $176.4(2)$ & & \\
$\mathrm{As}-\operatorname{Re}-\mathrm{N}(6)$ & $90.8(2)-97.4(2)$ & & \\
$\mathrm{As}-\mathrm{Re}-\mathrm{Cl}(5)$ & $79.07(7)-92.71(7)$ & & \\
\hline
\end{tabular}

Crystal data. $\mathrm{C}_{52} \mathrm{H}_{48} \mathrm{As}_{2} \mathrm{ClO}_{6} \mathrm{P}_{2} \mathrm{Re}, M_{\mathrm{r}}=1202.4$, monoclinic, space group $C c, a=9.868(3), b=22.608(5), c=$ $21.887(8) \AA, \beta=91.87(3)^{\circ}, U=4880(3) \AA^{3}, Z=4, D_{\mathrm{c}}=$ $1.637 \mathrm{~g} \mathrm{~cm}^{-3}, F(000)=2384$, crystal dimensions $=0.30 \times$ $0.40 \times 0.40 \mathrm{~mm}$. The intensity data were measured on a CAD-4 diffractometer using Mo-K $\alpha$ radiation $(\lambda=0.7107 \AA)$ using the $\omega-2 \theta$ scan mode at $297 \mathrm{~K}$ with $2 \theta_{\max }=45^{\circ}$. Cell dimensions were obtained from 24 reflections with $2 \theta$ angles in the range 18.72 22.54 3409 Reflections were measured of which 2822 were observed $[I>2.0 \sigma(I)]$. Absorption corrections $(\mu=33.1$ $\mathrm{cm}^{-1}$ ) were made according to $\psi$ curves of three selected reflections. The minimum and maximum transmission factors are 0.83 and 1.00. The structure was solved by the Patterson method and refined by least squares. Full-matrix least-squares refinement on 576 parameters converged to yield agreement indices $R=0.034, R^{\prime}=0.024$ and goodness of fit $=1.63 ; \max$. $\Delta / \sigma=0.34 ; \max . \Delta \rho=1.03$, min. $\Delta \rho=-0.75$ e $\AA^{-3}$; weights are based on counting statistics. Table 3 lists the atomic coordinates of non-hydrogen atoms. Selected bond distances and angles are summarized in Table 4. All hydrogen atom parameters are from calculated values. There are partial disorders on the $\mathrm{P}$ and As sites; the final result is based on $80 \%$ of As plus $20 \% \mathrm{P}$ for the As site and $80 \% \mathrm{P}$ plus $20 \%$ As for the $\mathrm{P}$ site.

Additional material available for both structures from the Cambridge Crystallographic Data Centre comprises $\mathrm{H}$-atom coordinates, thermal parameters and remaining bond lengths and angles.

\section{Results and Discussion}

Reaction of $\left[\mathrm{ReNCl}_{2}\left(\mathrm{PPh}_{3}\right)_{2}\right]$ with the appropriate diphosphines or arsines afforded the complex cations $\left[\mathrm{ReNL}_{2} \mathrm{Cl}\right]^{+}$in good yields, similar to that reported by Johnson. ${ }^{36}$ In the syntheses of the trans- $\left[\mathrm{ReO}_{2} \mathrm{~L}_{2}\right]^{+}$complexes, $\left[\mathrm{ReO}_{2}\left(\mathrm{PPh}_{3}\right)_{2} \mathrm{I}\right]$ has proven to be a good starting material for complexes of the $\mathrm{ReO}_{2}{ }^{+}$unit, as suggested by Brewer and Gray. ${ }^{6}$ It seems that both $\left[\mathrm{ReNCl}_{2}\left(\mathrm{PPh}_{3}\right)_{2}\right]$ and $\left[\mathrm{ReO}_{2}\left(\mathrm{PPh}_{3}\right)_{2} \mathrm{I}\right]$ are ideal starting materials for the general synthesis of the respective $\operatorname{Re}^{\mathrm{v}} \equiv \mathrm{N}$ and $\mathrm{O}=\mathrm{Re}^{\mathrm{v}}=\mathrm{O}$ units.

Fig. 1 shows the perspective drawing of the $\left[\mathrm{ReN}(\mathrm{dpae}){ }_{2} \mathrm{Cl}\right]^{+}$ cations. The molecule is essentially a distorted octahedron, with the perchlorate ions being non-co-ordinating. The As(1)-Re$\operatorname{As}(2)$ and $\mathrm{As}(3)-\operatorname{Re}-\mathrm{As}(4)$ bond angles are 80.86(4) and $81.04(4)^{\circ}$, respectively, as would be required by the bite distance of the chelating ligand. The nitrido ligand is trans to the chloro group with a $\mathrm{Cl}-\mathrm{Re}-\mathrm{N}$ angle of $176.4(2)^{\circ}$. The nitrido group exerts a large trans labilizing effect on the $\mathrm{Re}-\mathrm{Cl}$ bond [2.451(3) $\AA$ ] which is longer than that for a normal $\mathrm{Re}-\mathrm{Cl}$ bond though shorter than that reported for the trans $\mathrm{Re}-\mathrm{Cl}$ bond in $\left[\operatorname{ReNCl}_{2}\left(\mathrm{PEt}_{2} \mathrm{Ph}\right)_{3}\right] .^{7}$ The $\mathrm{Re} \equiv \mathrm{N}$ bond distance of $1.839(8) \AA$ is longer than that reported for other nitridorhenium( $(v)$ complexes such as $\left.\left[\mathrm{ReNCl}_{2}\left(\mathrm{PEt}_{2} \mathrm{Ph}\right)_{3}\right][d(\mathrm{Re} \equiv \mathrm{N})=1.79 \AA]\right]^{7}$ The shorter $\mathrm{Re}-\mathrm{Cl}$ and the longer $\mathrm{Re} \equiv \mathrm{N}$ bond distances than in $\left[\mathrm{ReNCl}_{2}\left(\mathrm{PEt}_{2} \mathrm{Ph}\right)_{3}\right]^{7}$ may not be a genuine representation and may arise as a result of crystallographic effects. ${ }^{8}$ The other bond distances and angles are normal. 
Table 3 Atomic parameters for trans- $\left[\mathrm{ReO}_{2}(\text { dadpe })_{2}\right] \mathrm{ClO}_{4}$ with e.s.d.s in parentheses

\begin{tabular}{|c|c|c|c|c|c|c|c|}
\hline Atom & $x$ & $y$ & $z$ & Atom & $x$ & $y$ & $z$ \\
\hline $\mathrm{Re}$ & 0.95138 & $0.79813(3)$ & 0.95495 & $C(24 B)$ & $1.4565(16)$ & $0.6647(8)$ & $0.8939(7)$ \\
\hline $\operatorname{As}(1)$ & $0.8439(2)$ & $0.8219(1)$ & $1.0568(1)$ & $C(25 B)$ & $1.3435(17)$ & $0.6371(8)$ & $0.8855(7)$ \\
\hline $\operatorname{As}(2)$ & $1.0594(2)$ & $0.7725(1)$ & $0.8545(1)$ & $\mathrm{C}(26 \mathrm{~B})$ & $1.2228(15)$ & $0.6691(6)$ & $0.8717(6)$ \\
\hline$P(3)$ & $0.9620(3)$ & $0.6995(1)$ & $1.0026(1)$ & $C(31 \mathrm{~A})$ & $1.1102(14)$ & $0.6838(6)$ & $1.0525(6)$ \\
\hline$P(4)$ & $0.9588(3)$ & $0.9011(1)$ & $0.9048(1)$ & $C(32 A)$ & $1.0987(16)$ & $0.6524(7)$ & $1.1074(6)$ \\
\hline$O(5)$ & $1.1148(9)$ & $0.8217(4)$ & $0.9826(4)$ & $C(33 A)$ & $1.2129(16)$ & $0.6417(8)$ & $1.1438(6)$ \\
\hline$O(6)$ & $0.7866(8)$ & $0.7801(4)$ & $0.9270(3)$ & $C(34 A)$ & $1.3346(15)$ & $0.6626(7)$ & $1.1272(7)$ \\
\hline$C(1)$ & $0.8000(13)$ & $0.7466(5)$ & $1.0934(6)$ & $C(35 A)$ & $1.3472(14)$ & $0.6929(7)$ & $1.0740(6)$ \\
\hline$C(2)$ & $1.1005(18)$ & $0.8470(7)$ & $0.8167(7)$ & $C(36 A)$ & $1.2355(13)$ & $0.7039(7)$ & $1.0380(5)$ \\
\hline$C(3)$ & $0.8116(13)$ & $0.6978(7)$ & $1.051 \mathrm{I}(5)$ & $C(31 B)$ & $0.9396(18)$ & $0.6359(5)$ & $0.9529(7)$ \\
\hline$C(4)$ & $1.1134(15)$ & $0.8966(6)$ & $0.8595(6)$ & $C(32 B)$ & $1.0042(13)$ & $0.5828(16)$ & $0.9620(6)$ \\
\hline$C(11 \mathrm{~A})$ & $0.9397(13)$ & $0.8618(6)$ & $1.1200(5)$ & $C(33 B)$ & $0.9882(16)$ & $0.5353(6)$ & $0.9246(7)$ \\
\hline$C(12 A)$ & $1.0380(14)$ & $0.9032(6)$ & $1.1057(6)$ & $C(34 B)$ & $0.9028(16)$ & $0.5390(6)$ & $0.8753(7)$ \\
\hline$C(13 A)$ & $1.1042(15)$ & $0.9372(7)$ & $1.1496(6)$ & $C(35 B)$ & $0.8350(14)$ & $0.5932(7)$ & $0.8630(6)$ \\
\hline$C(14 A)$ & $1.0728(16)$ & $0.9279(7)$ & $1.2085(7)$ & $C(36 B)$ & $0.8548(14)$ & $0.6399(6)$ & $0.9020(6)$ \\
\hline$C(15 A)$ & $0.9818(17)$ & $0.8856(8)$ & $1.2258(6)$ & $C(41 A)$ & $0.8309(15)$ & $0.9253(6)$ & $0.8511(6)$ \\
\hline$C(16 A)$ & $0.9165(15)$ & $0.8527(7)$ & $1.1820(6)$ & $C(42 A)$ & $0.7054(17)$ & $0.8988(7)$ & $0.8510(6)$ \\
\hline$C(11 B)$ & $0.6743(14)$ & $0.8629(6)$ & $1.0545(5)$ & $C(43 A)$ & $0.5975(18)$ & $0.9176(8)$ & $0.8076(8)$ \\
\hline$C(12 B)$ & $0.5640(14)$ & $0.8376(7)$ & $1.0218(7)$ & $\mathrm{C}(44 \mathrm{~A})$ & $0.6207(18)$ & $0.9625(7)$ & $0.7698(7)$ \\
\hline$C(13 B)$ & $0.4408(14)$ & $0.8640(8)$ & $1.0199(8)$ & $C(45 A)$ & $0.7471(20)$ & $0.9874(9)$ & $0.7697(8)$ \\
\hline$C(14 B)$ & $0.4233(16)$ & $0.9173(8)$ & $1.0478(7)$ & $C(46 A)$ & $0.8496(17)$ & $0.9689(8)$ & $0.8110(7)$ \\
\hline$C(15 B)$ & $0.5306(16)$ & $0.9466(7)$ & $1.0807(7)$ & $C(41 B)$ & $0.9848(13)$ & $0.9649(5)$ & $0.9525(7)$ \\
\hline$C(16 B)$ & $0.6546(14)$ & $0.9175(6)$ & $1.0809(6)$ & $C(42 B)$ & $0.8828(13)$ & $0.9913(6)$ & $0.9811(5)$ \\
\hline$C(21 \mathrm{~A})$ & $0.9620(14)$ & $0.7351(6)$ & $0.7880(5)$ & $\mathrm{C}(43 \mathrm{~B})$ & $0.8978(16)$ & $1.0370(6)$ & $1.0225(7)$ \\
\hline$C(22 A)$ & $1.0253(15)$ & $0.7015(8)$ & $0.7445(6)$ & $C(44 B)$ & $1.0253(17)$ & $1.0568(6)$ & $1.0351(6)$ \\
\hline$C(23 A)$ & $0.9510(21)$ & $0.6775(8)$ & $0.6966(7)$ & $\mathrm{C}(45 \mathrm{~B})$ & $1.1316(15)$ & $1.0338(6)$ & $1.0076(7)$ \\
\hline$C(24 A)$ & $0.8143(18)$ & $0.6925(9)$ & $0.6897(6)$ & $C(46 B)$ & $1.1063(16)$ & $0.9890(6)$ & $0.9652(6)$ \\
\hline$C(25 A)$ & $0.7547(16)$ & $0.7228(8)$ & $0.7329(6)$ & $\mathrm{Cl}$ & $0.2746(4)$ & $0.9144(2)$ & $0.6919(2)$ \\
\hline$C(26 A)$ & $0.8251(15)$ & $0.7465(7)$ & $0.7813(6)$ & $O(1)$ & $0.3328(10)$ & $0.9407(5)$ & $0.6408(4)$ \\
\hline$C(21 B)$ & $1.2283(14)$ & $0.7284(6)$ & $0.8655(5)$ & $\mathrm{O}(2)$ & $0.1388(12)$ & $0.9018(6)$ & $0.6761(5)$ \\
\hline$C(22 B)$ & $1.3476(16)$ & $0.7572(7)$ & $0.8741(7)$ & $O(3)$ & $0.2751(11)$ & $0.9541(5)$ & $0.7410(4)$ \\
\hline$C(23 B)$ & $1.4661(15)$ & $0.7233(8)$ & $0.8877(7)$ & $\mathrm{O}(4)$ & $0.3469(11)$ & $0.8621(5)$ & $0.7085(4)$ \\
\hline
\end{tabular}

Table 4 Selected bond distances $(\AA)$ and angles $\left({ }^{\circ}\right)$ for trans$\left[\mathrm{ReO}_{2}(\text { dadpe })_{2}\right]^{+}$with e.s.d.s in parentheses

\begin{tabular}{llll}
$\operatorname{Re}-\mathrm{O}(5)$ & $1.785(8)$ & $\mathrm{Re}-\mathrm{O}(6)$ & $1.766(8)$ \\
$\operatorname{Re}-\mathrm{As}(1)$ & $2.557(2)$ & $\operatorname{Re}-\mathrm{As}(2)$ & $2.541(2)$ \\
$\operatorname{Re}-\mathrm{P}(3)$ & $2.463(3)$ & $\operatorname{Re}-\mathrm{P}(4)$ & $2.576(3)$ \\
$\mathrm{As}(1)-\mathrm{C}(1)$ & $1.94(1)$ & $\mathrm{As}(2)-\mathrm{C}(2)$ & $1.93(2)$ \\
$\mathrm{P}(3)-\mathrm{C}(3)$ & $1.85(1)$ & $\mathrm{P}(4)-\mathrm{C}(4)$ & $1.85(1)$ \\
$\mathrm{As}-\mathrm{C}(\mathrm{Ph})$ & $1.88(1)-1.95(1)$ & & \\
$\mathrm{P}-\mathrm{C}(\mathrm{Ph})$ & $1.78(2)-1.83(1)$ & & \\
$\mathrm{O}(5)-\operatorname{Re}-\mathrm{O}(6)$ & $176.0(4)$ & $\mathrm{P}(3)-\operatorname{Re}-\mathrm{P}(4)$ & $175.93(9)$ \\
$\mathrm{As}(1)-\operatorname{Re}-\mathrm{As}(2)$ & $178.88(6)$ & $\mathrm{As}(2)-\operatorname{Re}-\mathrm{P}(4)$ & $79.57(7)$ \\
$\mathrm{As}(1)-\operatorname{Re}-\mathrm{P}(3)$ & $80.45(8)$ & $\mathrm{As}(2)-\operatorname{Re}-\mathrm{P}(3)$ & $98.44(8)$ \\
$\mathrm{As}(1)-\operatorname{Re}-\mathrm{P}(4)$ & $101.52(7)$ & $\mathrm{As}(1)-\operatorname{Re}-\mathrm{O}(6)$ & $86.95(25)$ \\
$\mathrm{As}(1)-\operatorname{Re}-\mathrm{O}(5)$ & $92.2(3)$ & $\mathrm{As}(2)-\operatorname{Re}-\mathrm{O}(6)$ & $93.19(25)$ \\
$\mathrm{As}(2)-\operatorname{Re}-\mathrm{O}(5)$ & $87.7(3)$ & $\mathrm{P}(3)-\operatorname{Re}-\mathrm{O}(6)$ & $87.9(3)$ \\
$\mathrm{P}(3)-\operatorname{Re}-\mathrm{O}(5)$ & $95.8(3)$ & $\mathrm{P}(4)-\operatorname{Re}-\mathrm{O}(6)$ & $95.7(3)$ \\
$\mathrm{P}(4)-\operatorname{Re}-\mathrm{O}(5)$ & $80.6(3)$ & & \\
\hline
\end{tabular}

Fig. 2 shows the perspective drawing of the trans$\left[\mathrm{ReO}_{2}(\mathrm{dadpe})_{2}\right]^{+}$cation. The cation is a distorted octahedron, with the perchlorate ions being non-co-ordinating. The $\operatorname{As}(1)-\operatorname{Re}-\mathrm{As}(2)$ and $\mathrm{P}(3)-\operatorname{Re}-\mathrm{P}(4)$ bond angles are almost linear, being $178.88(6)$ and $175.93(9)^{\circ}$, respectively. The $P$ atoms on the two dadpe ligands are trans to each other, the same is true for the As atoms. The two oxo groups are trans to each other with a $\mathrm{O}(5)-\mathrm{Re}-\mathrm{O}(6)$ bond angle of $176.0(4)^{\circ}$. The $\mathrm{Re}=\mathrm{O}$ bond distances are $1.785(8)$ and $1.766(8) \AA$, which are in the normal range for trans-dioxorhenium(v) complexes. ${ }^{9}$ The other bond distances and angles are also normal.

All the nitridorhenium(v) and trans-dioxorhenium(v) complexes are air-stable solids. Magnetic susceptibility measurements show that they are diamagnetic, consistent with the $\left(\mathrm{d}_{x y}\right)^{2}$ ground-state electronic configuration in a $C_{2 v}$ and $D_{2 h}$ symmetry, respectively. A comparison between the IR absorp-

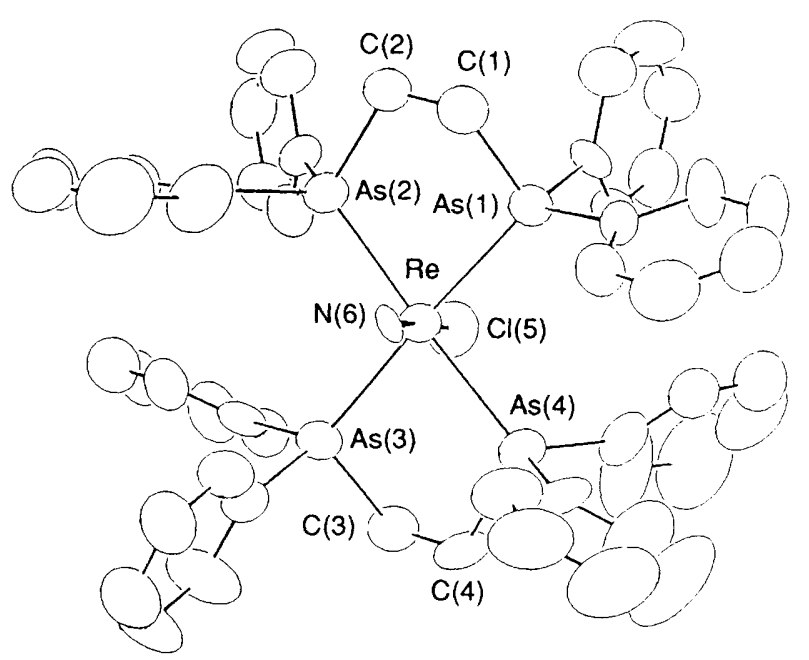

Fig. 1 A perspective drawing of the $\left[\operatorname{ReN}(\text { dpae })_{2} \mathrm{Cl}\right]^{+}$cation with atomic numbering

tion spectra of the corresponding nitrido- and dioxo-rhenium(v) complexes allows the assignment of the $\mathrm{Re} \equiv \mathrm{N}$ and $\mathrm{Re}=\mathrm{O}$ stretches to be made. All the nitridorhenium(v) complexes exhibit a strong IR absorption band at $c a$. 1043-1049 $\mathrm{cm}^{-1}$, assignable as the $\mathrm{Re} \equiv \mathrm{N}$ stretch. The observed stretching frequencies are comparable to those found for other nitridorhenium(v) complexes. ${ }^{3 a, b, 10}$ All the trans-dioxorhenium(v) complexes are found to exhibit a strong IR absorption band at $c a$. $785-790 \mathrm{~cm}^{-1}$, assignable to the $v_{\text {asym }}(\operatorname{Re}=\mathrm{O})$ stretch, typical of the trans- $\mathrm{ReO}_{2}$ unit.9.11

Both the nitrido and dioxo complexes are 1:1 electrolytes in acetonitrile and in methanol. The dissociation of the tran:- 


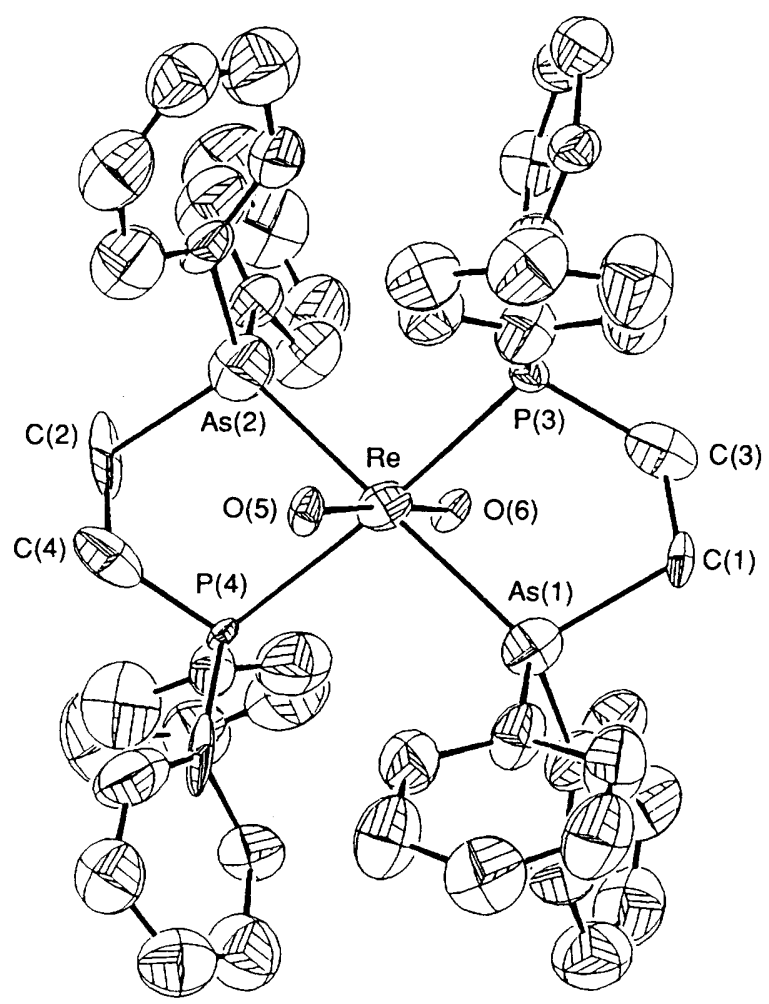

Fig. 2 A perspective drawing of the trans- $\left[\mathrm{ReO}_{2}(\text { dadpe })_{2}\right]^{+}$cation with atomic numbering

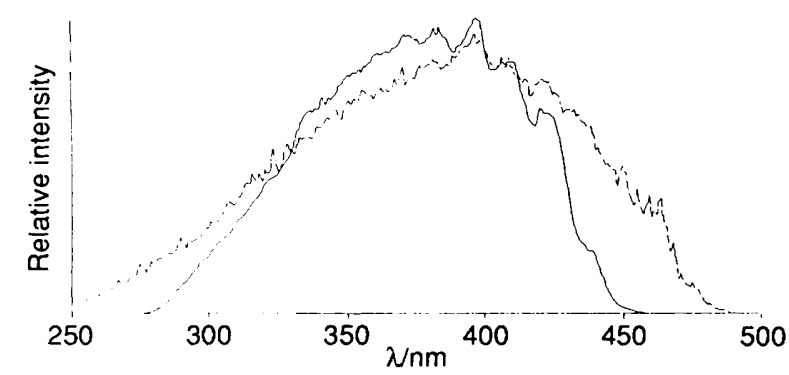

Fig. 3 Solid-state excitation spectra of $(a)\left[\mathrm{ReN}(R-1,2 \text {-dppp })_{2} \mathrm{Cl}_{\mathrm{ClO}}\right.$ $(-)$ and $(b)$ trans $-\left[\operatorname{ReO}_{2}(R-1,2-\mathrm{dppp})_{2}\right] \mathrm{ClO}_{4}(---)$ at $77 \mathrm{~K}$

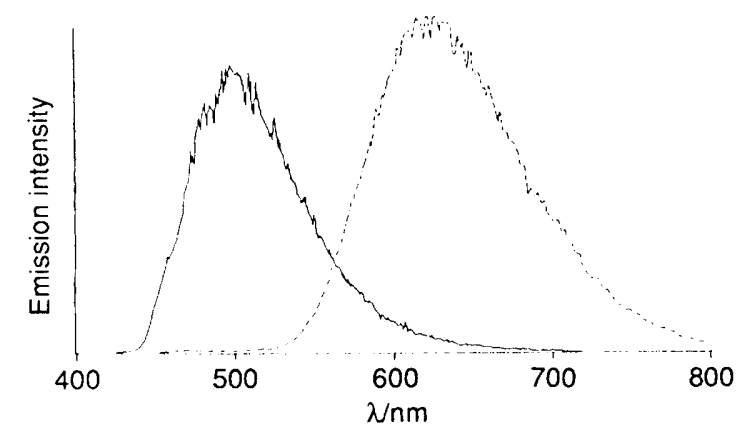

Fig. 4 Solid-state emission spectra of $(a)\left[\mathrm{ReN}(R-1,2-\mathrm{dppp})_{2} \mathrm{Cl}\right] \mathrm{ClO}_{4}$ $(--)$ and $(h)$ trans- $\left[\operatorname{ReO}_{2}(R-1,2-\mathrm{dppp})_{2}\right] \mathrm{ClO}_{4}(---)$ at $77 \mathrm{~K}$

chloro ligand in $\left[\mathrm{Re}^{\mathrm{v}} \mathrm{NL}_{2} \mathrm{Cl}\right]^{+}$appears to be unimportant in both $\mathrm{MeCN}$ and $\mathrm{MeOH}$, as evidenced from conductivity measurements and ${ }^{31} \mathrm{P}$ NMR spectroscopy. The presence of two ${ }^{31} \mathrm{P}$ NMR singlets for both $\left[\mathrm{Re}^{\mathrm{v}} \mathrm{N}(\text { dadpe }){ }_{2} \mathrm{Cl}\right]^{+}$and trans$\left[\mathrm{Re}^{\mathrm{V}} \mathrm{O}_{2}(\mathrm{dadpe})\right]^{+}$is consistent with the presence of two isomers, one with the As-donor atoms trans and the other cis to each other.

The UV/VIS absorption spectra of complexes [ $\left.\mathrm{ReNL}_{2} \mathrm{Cl}\right]$ $\mathrm{ClO}_{4}$ in acetonitrile exhibit intense absorption bands in the
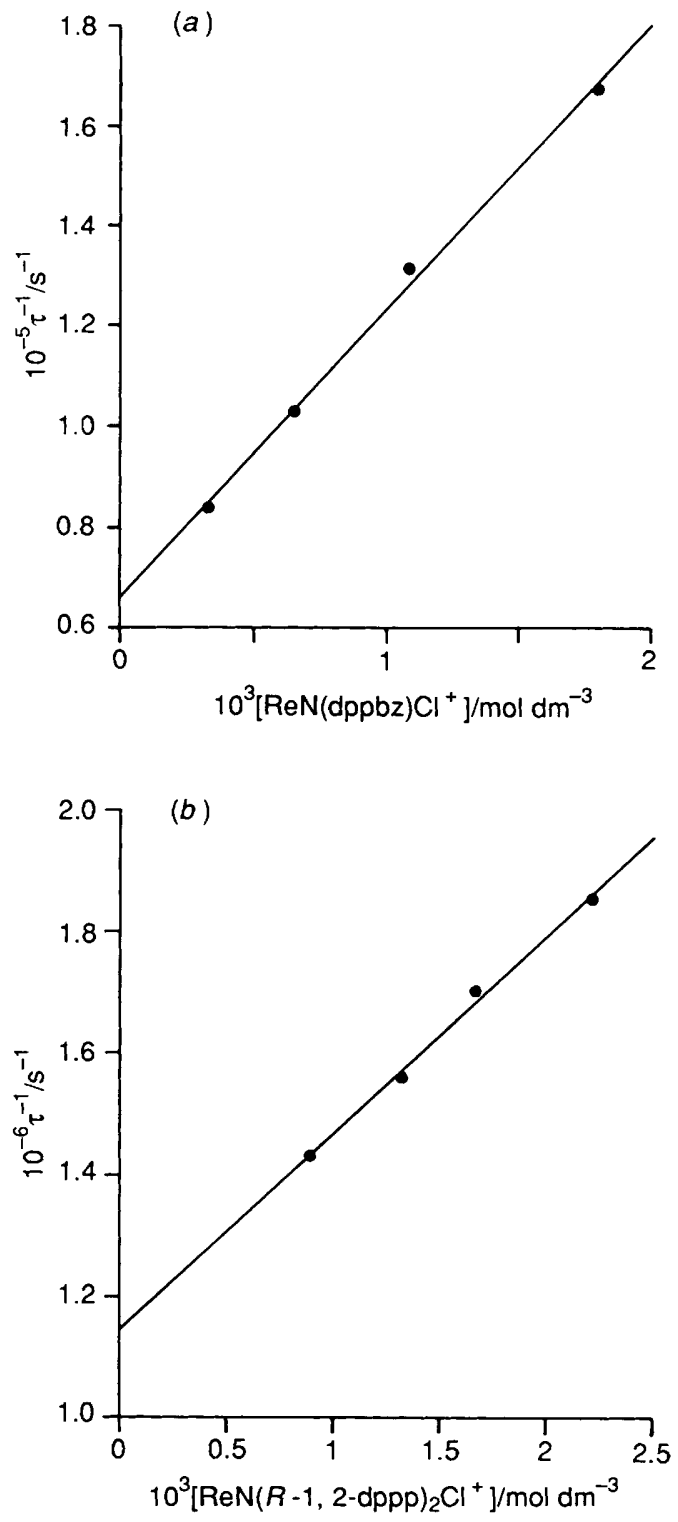

Fig. 5 Plot of $1 / \tau$ versus concentration of $(a)\left[\mathrm{ReN}(\mathrm{dppbz})_{2} \mathrm{Cl}_{\mathrm{ClO}} \mathrm{ClO}_{4}\right.$ and $(b)\left[\operatorname{ReN}(R-1,2-\mathrm{dppp})_{2} \mathrm{Cl}_{3} \mathrm{ClO}_{4}\right.$ in degassed acetonitrile $(0.1 \mathrm{~mol}$ $\left.\mathrm{dm}^{-3} \mathrm{NBu}_{4} \mathrm{PF}_{6}\right)$ at $25^{\circ} \mathrm{C}$

region $220-300 \mathrm{~nm}$ and a weak absorption at ca. $360-375 \mathrm{~nm}$ $\left(\varepsilon \approx 250-330 \mathrm{dm}^{3} \mathrm{~mol}^{-1} \mathrm{~cm}^{-1}\right.$ ) with very weak shoulders in the region $400-550 \mathrm{~nm}(\varepsilon<100)$. The large absorption coefficients observed for the bands at $c a .200-300 \mathrm{~nm}$ possibly suggest their origins as intraligand transition and ligand-to-metal chargetransfer transitions $\left[\mathrm{p}_{\pi}\left(\mathrm{N}^{3-}\right) \longrightarrow \mathrm{d}_{\pi^{*}}(\mathrm{Re})\right.$ and $\mathrm{p}_{\pi}\left(\mathrm{Cl}^{-}\right) \longrightarrow \mathrm{d}_{\pi^{*}}$ (Re)] where $d_{\pi^{*}}=d_{x z}, d_{y z}$. The relative insensitivity of the transition energies of these intense absorptions to the nature of the equatorial ligands probably rules out their assignment as metal-to-ligand charge-transfer transitions $\left[\mathrm{d}_{x y} \longrightarrow \pi^{*}(\mathrm{~L})\right]$. With reference to previous spectroscopic works on $\mathrm{d}^{2}$ nitridoosmium(V1) systems, ${ }^{2}$ the energies of $d$ orbitals of $\mathrm{Re}^{\mathrm{v}} \equiv \mathrm{N}$ complexes, assuming a $C_{2 v}$ symmetry are in the order, $\mathrm{a}_{2}$ $\left(\mathrm{d}_{x y}\right)<\mathrm{b}_{1}\left(\mathrm{~d}_{x z}\right) \approx \mathrm{b}_{2}\left(\mathrm{~d}_{y z}\right)<\mathrm{a}_{1}\left(\mathrm{~d}_{x^{2}-y^{2}}\right)<\mathrm{a}_{1}\left(\mathrm{~d}_{z^{2}}\right)$. The weak absorptions in the low-energy region probably arise from transitions to excited states derived from spin triplets $\left[\left(\mathrm{d}_{x y}\right)^{2}\right.$ $\longrightarrow\left(\mathrm{d}_{x y}\right)^{1}\left(\mathrm{~d}_{y z}\right)^{1}\left({ }^{1} \mathrm{~A}_{1} \longrightarrow{ }^{3} \mathrm{~B}_{1}\right)$ and $\left(\mathrm{d}_{x y}\right)^{2} \longrightarrow\left(\mathrm{d}_{x y}\right)^{1}\left(\mathrm{~d}_{x z}\right)^{1}$ $\left({ }^{1} \mathrm{~A}_{1} \longrightarrow\left(\mathrm{d}_{x y} \mathrm{~B}_{2}\right)\right]$.

The UV/VIS absorption spectra of complexes trans$\left[\mathrm{ReO}_{2} \mathrm{~L}_{2}\right] \mathrm{ClO}_{4}$ in acetonitrile are also dominated by intense absorptions in the high-energy region, which are likely to be intraligand and charge-transfer $\left[p_{\pi}\left(O^{2-}\right) \longrightarrow\left(d_{x z}, d_{y z}\right)(R e)\right]$ 
Table 5 Photophysical data for complexes $\left[\mathrm{ReNL}_{2} \mathrm{Cl}\right] \mathrm{ClO}_{4}(a)$ and trans- $\left[\mathrm{ReO}_{2} \mathrm{~L}_{2}\right] \mathrm{ClO}_{4}(b)$ at room temperature

(a) $\left[\mathrm{ReNL}{ }_{2} \mathrm{Cl}\right] \mathrm{ClO}_{4}$

$\begin{array}{ll}\mathrm{L} & \lambda_{\mathrm{em}} / \mathrm{nm}\left(\tau_{\mathrm{o}} / \mu \mathrm{s}\right) \\ \mathrm{dppe} & 512(2.0 \pm 0.2)^{a} \\ \text { dppbz } & 514(0.35 \pm 0.05,1.7 \pm 0.2)^{a} \\ & 509(10 \pm 1)^{b} \\ & 509(10 \pm 1)^{c} \\ \text { dpae } & 509^{d} \\ \text { dadpe } & 500(0.45 \pm 0.05)^{a} \\ R-1,2 \text {-dppp } & 510(0.50 \pm 0.05,2.7 \pm 0.3)^{a} \\ & 502(9.5 \pm 1.0)^{a} \\ & 520(0.7 \pm 0.1)^{b} \\ & 520(0.7 \pm 0.1)^{c} \\ & 519^{d}\end{array}$

(b) trans- $\left[\mathrm{ReO}_{2} \mathrm{~L}_{2}\right] \mathrm{ClO}_{4}$

$\begin{array}{ll}\mathrm{L} & \lambda_{\mathrm{cm}} / \mathrm{nm}\left(\tau_{\mathrm{o}} / \mu \mathrm{s}\right) \\ \text { dppe } & 613(0.20 \pm 0.2,1.2 \pm 0.2)^{a} \\ & 615(0.8 \pm 0.1)^{b} \\ \text { dppbz } & 607(0.7 \pm 0.1)^{a} \\ & 627(1.0 \pm 0.2)^{b} \\ \text { dpae } & 596(1.5 \pm 0 .)^{a} \\ & 627(0.8 \pm 0.1)^{b} \\ \text { dadpe } & 618(0.20 \pm 0.02,1.4 \pm 0.2)^{a} \\ & 630(0.5 \pm 0.1)^{b} \\ R \text {-1,2-dppp } & 613(0.35 \pm 0.05)^{a} \\ & 620(0.7 \pm 0.1)^{b}\end{array}$

${ }^{a}$ Solid-state emission. Some show biexponential decays. ${ }^{b}$ In degassed $\mathrm{MeCN}\left(0.1 \mathrm{~mol} \mathrm{dm}^{-3} \mathrm{NBu}_{4} \mathrm{PF}_{6}\right) \cdot 0.65 \mathrm{mmol} \mathrm{dm}^{-3}=\left[\mathrm{ReNL}_{2} \mathrm{Cl}\right] \mathrm{ClO}_{4}$; $3.5 \mathrm{mmol} \mathrm{dm}^{-3}=\left[\mathrm{ReO}_{2} \mathrm{~L}_{2}\right] \mathrm{ClO}_{4}$. The lifetimes measured for the dioxo species in $\mathrm{MeCN}$ are not very accurate due to the very weak emission signal detected. ${ }^{c}$ In degassed $\mathrm{MeOH}-\mathrm{MeCN}(1: 1 \mathrm{v} / \mathrm{v}) .{ }^{d}$ In degassed $\mathrm{CH}_{2} \mathrm{Cl}_{2}$.

Table 6 Cyclic voltammetric data for complexes $\left[\mathrm{ReNL}_{2} \mathrm{Cl} \mathrm{ClO}_{4}(a)\right.$ and trans- $\left[\mathrm{ReO}_{2} \mathrm{~L}_{2}\right] \mathrm{ClO}_{4}(b)$ in acetonitrile $\left(0.1 \mathrm{~mol} \mathrm{dm}^{-3} \mathrm{NBu}_{4} \mathrm{PF}_{6}\right)^{a}$

(a) $\left[\mathrm{ReNL}_{2} \mathrm{Cl}\right] \mathrm{ClO}_{4}$

$\begin{array}{lll}\mathrm{L} & E_{\mathrm{pc}} / \mathrm{V}^{b} & E_{\mathrm{pa}} / \mathrm{V}^{c} \\ \text { dppe } & -2.34 & +1.55 \\ \text { dppbz } & -2.26 & +1.57 \\ \text { dpae } & -2.21 & +1.65 \\ \text { dadpe } & -2.17 & +1.51 \\ R \text {-1,2-dppp } & -2.36 & +1.45\end{array}$

(b) trans- $\left[\mathrm{ReO}_{2} \mathrm{~L}_{2}\right] \mathrm{ClO}_{4}$

$\begin{array}{lll}\text { dppe } & -2.27 & +1.33 \\ & -2.39 & \\ \text { dppbz } & -2.26 & +1.43 \\ & -2.37 & \\ \text { dpae } & -2.13 & +1.33 \\ & -2.31 & \\ \text { dadpe } & -2.44 & +1.43 \\ R \text {-1,2-dppp } & -2.26 & +1.30 \\ & -2.35 & \end{array}$

${ }^{a}$ Working electrode, glassy carbon; scan rate; $100 \mathrm{mV} \mathrm{s}^{-1}$; potentials quoted relative to the ferrocene-ferrocenium couple. ${ }^{b} E_{\mathrm{pc}}$ is the peak cathodic potential for the irreversible couple. ${ }^{C} E_{\mathrm{pa}}$ is the peak anodic potential for the irreversible couple.

transitions. With reference to previous spectroscopic works on $\mathrm{d}^{2}$ trans-dioxorhenium(v) systems, ${ }^{1 a, b}$ the energies of d orbitals of $\mathrm{O}=\mathrm{Re}^{\mathrm{v}}=\mathrm{O}$ complexes, assuming a $D_{2 h}$ symmetry are in the order, $b_{1 \mathrm{~g}}\left(\mathrm{~d}_{x y}\right)<\mathrm{b}_{2 \mathrm{~g}}\left(\mathrm{~d}_{x z}\right) \approx \mathrm{b}_{3 \mathrm{~g}}\left(\mathrm{~d}_{y z}\right)<\mathrm{a}_{\mathrm{g}}\left(\mathrm{d}_{x^{2}-y^{2}}\right)<\mathrm{a}_{\mathrm{g}}$ $\left(d_{z^{2}}\right)$. The very weak absorptions that tail into the $390-500 \mathrm{~nm}$ region are assigned as the $\left(\mathrm{d}_{x y}\right)^{2} \longrightarrow\left(\mathrm{d}_{x y}\right)^{1}\left(\mathrm{~d}_{x z}\right)^{1}\left[{ }^{1} \mathrm{~A}_{\mathrm{g}} \longrightarrow\right.$
$\left.{ }^{3} \mathrm{~B}_{3 \mathrm{~g}}\right]$ and $\left(\mathrm{d}_{x y}\right)^{2} \longrightarrow\left(\mathrm{d}_{x y}\right)^{1}\left(\mathrm{~d}_{y z}\right)^{1}\left[{ }^{1} \mathrm{~A}_{\mathrm{g}} \longrightarrow{ }^{3} \mathrm{~B}_{2 \mathrm{~g}}\right]$ transitions

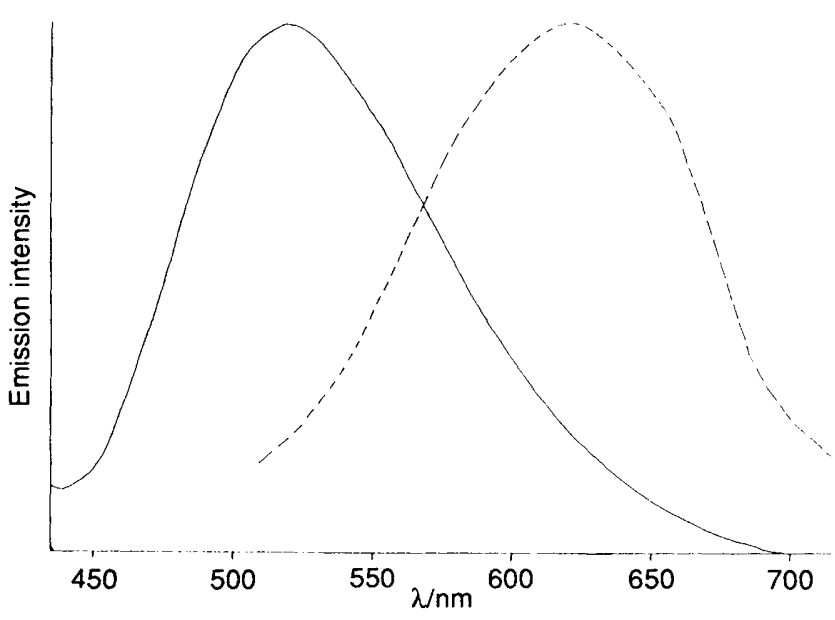

Fig. 6 Emission spectra of $(a)\left[\mathrm{ReN}(R-1,2-\mathrm{dppp})_{2} \mathrm{Cl} \mathrm{ClO}_{4}(-)\right.$ and (b) trans-[ $\left[\mathrm{ReO}_{2}(R-1,2-\mathrm{dppp})_{2}\right] \mathrm{ClO}_{4}(--\longrightarrow)$ in degassed acetonitrile at $25^{\circ} \mathrm{C}$

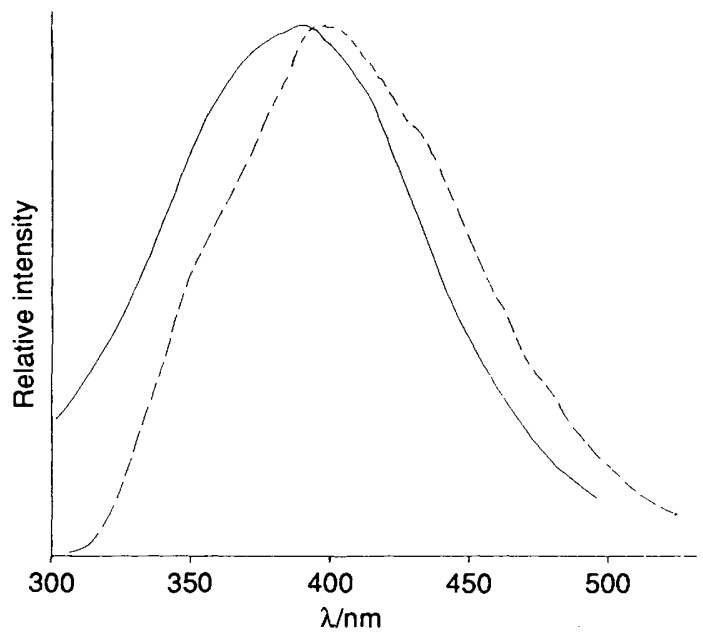

Fig. 7 Excitation spectra of $(a)\left[\mathrm{ReN}(R-1,2-\mathrm{dppp})_{2} \mathrm{Cl}\right] \mathrm{ClO}_{4}(-)$ and (b) trans- $\left[\mathrm{ReO}_{2}(R-1,2-\mathrm{dppp})_{2}\right] \mathrm{ClO}_{4}(-\longrightarrow)$ in degassed acetonitrile at $25^{\circ} \mathrm{C}$

which are vibronically coupled to the $\mathrm{B}_{1 \mathrm{u}}\left[\mathrm{ReO}_{2}\right]$ stretching mode.

Excitation of solid samples of complexes $\left[\mathrm{ReNL}_{2} \mathrm{Cl}_{3} \mathrm{ClO}_{4}\right.$ and trans- $\left[\mathrm{ReO}_{2} \mathrm{~L}_{2}\right] \mathrm{ClO}_{4}$ at $350-380 \mathrm{~nm}$ at room temperature results in yellow-green and orange emission, respectively. The solid-state excitation and emission spectra at $77 \mathrm{~K}$ are depicted in Figs. 3 and 4, respectively. Well resolved vibronic structures are observed in the solid-state excitation spectrum of $[\operatorname{Re}(\mathrm{N})(R$ 1,2-dppp) $\left.{ }_{2} \mathrm{Cl}\right] \mathrm{ClO}_{4}$ at $77 \mathrm{~K}$ with a progression spacing of $c a$. $750-850 \mathrm{~cm}^{-1}$. Similar progressions have been observed in the low-temperature absorption spectrum of $\left[\mathrm{OsNCl}_{4}\right]^{-},{ }^{2 a, b}$ suggestive of a weakened $\mathbf{M} \equiv \mathbf{N}$ bond in the excited state. The photophysical data are summarized in Table 5 . Both $\left[\mathrm{ReN}(\mathrm{dppbz}){ }_{2} \mathrm{Cl}\right]^{+}$and $\left[\mathrm{Re}(\mathrm{N})(R-1,2-\mathrm{dppp})_{2} \mathrm{Cl}\right]^{+}$exhibit intense long-lived room-temperature luminescence in fluid solutions while only a very weak emission at $c a .500 \mathrm{~nm}$ is detected in fluid solutions of $\left[\mathrm{ReN}(\mathrm{dppe})_{2} \mathrm{Cl}^{+}\right.$. Concentration quenching has been observed for both $\left[\mathrm{ReN}(\mathrm{dppbz})_{2} \mathrm{Cl}^{+}\right.$and $\left[\operatorname{ReN}(R-1,2-\mathrm{dppp})_{2} \mathrm{Cl}^{+}\right.$in $\mathrm{MeCN}\left(0.1 \mathrm{~mol} \mathrm{dm}{ }^{-3} \mathrm{NBu}_{4} \mathrm{PF}_{6}\right)$ with self-quenching rate constants of $\mathrm{ca} .5 .7 \times 10^{7}$ and $3.2 \times 10^{8} \mathrm{dm}^{3} \mathrm{~mol}^{-1} \mathrm{~s}^{-1}$, respectively, and inherent lifetimes of ca. 15 and $0.9 \mu \mathrm{s}$ at infinite dilution (Fig. 5). A possible mechanism for self quenching would involve the coupling of two $\mathrm{Re} \equiv \mathrm{N}$ units as suggested by Che et $_{\text {al }}{ }^{2 \mathrm{c}}$ in the analogous $\mathrm{Os}{ }^{\mathrm{v}} \equiv \mathrm{N}$ system. However, further studies have to be followed in order to elucidate the mechanism of concentration quenching. The much 
shorter excited-state lifetime of $\left[\mathrm{ReN}(\mathrm{dppbz})_{2} \mathrm{Cl}\right]^{+}$in the solid state than in acetonitrile is rather surprising. On the other hand, all the trans- $\left[\mathrm{ReO}_{2} \mathrm{~L}_{2}\right]^{+}$complexes exhibit room-temperature luminescence in $\mathrm{MeCN}$. The emission of these dioxo species is found to be completely quenched in $\mathrm{MeOH}$, similar to that reported previously by Winkler and Gray, ${ }^{1 a, b}$ where interaction of the $\mathrm{M}=\mathrm{O}$ unit with the $\mathrm{OH}$ group of the solvent has been suggested to provide an efficient relaxation pathway for the excited species. However, the lifetimes of both $\left[\operatorname{ReN}(\mathrm{dppbz})_{2}-\right.$ $\mathrm{Cl}]^{+}$and $\left[\operatorname{ReN}(R-1,2-\mathrm{dppp})_{2} \mathrm{Cl}\right]^{+}$are found to be rather difference in the $\mathrm{p} K_{\mathrm{a}}$ values of the nitrido and oxo group. The room-temperature emission and excitation spectra of $\left[\mathrm{ReNL}_{2}-\right.$ $\mathrm{Cl}^{+}$and trans- $\left[\mathrm{ReO}_{2} \mathrm{~L}_{2}\right]^{+}$in degassed acetonitrile are shown in Figs. 6 and 7 , respectively. The excitation spectra of $\left[\mathrm{ReNL}_{2} \mathrm{Cl}\right]^{+}$show broad bands at ca. $390-410 \mathrm{~nm}$. This together with the observed lifetime in the microsecond range suggest that the emissive state is likely to be derived from the $\left(\mathrm{d}_{x y}\right)^{1}\left(\mathrm{~d}_{x z}\right)^{1}$ and $\left(\mathrm{d}_{x y}\right)^{1}\left(\mathrm{~d}_{y z}\right)^{1}$ triplets. The excitation spectra of trans- $\left[\mathrm{ReO}_{2} \mathrm{~L}_{2}\right]^{+}$show bands at ca. $400-440 \mathrm{~nm}$, probably also derived from the $\left(\mathrm{d}_{x y}\right)^{1}\left(\mathrm{~d}_{x z}\right)^{1}\left[{ }^{3} \mathrm{~B}_{3 \mathrm{~g}}\right]$ and $\left(\mathrm{d}_{x y}\right)^{1}\left(\mathrm{~d}_{y z}\right)^{1}\left[{ }^{3} \mathrm{~B}_{2 \mathrm{~g}}\right]$ triplets assuming a $D_{2 h}$ symmetry. Similar assignments have also been made for other $\mathrm{d}^{2}$ nitrido and dioxo systems ${ }^{1.2}$ where the $\left(\mathrm{d}_{x y}\right)^{1}\left(\mathrm{~d}_{x z}, \mathrm{~d}_{y z}\right)^{1}$ triplet $\left({ }^{3} \mathrm{E}\right.$ in $C_{4 v}$ and ${ }^{3} \mathrm{E}_{\mathrm{g}}$ in $D_{4 h}$ symmetry) is suggested to be the origin of the observed emission.

An interesting feature worth mentioning is that a comparison between the emission energies of the nitrido- and the transdioxo-rhenium(v) complexes shows that those of the former occur at higher energies than the latter. This probably indicates that the nitrido group is a better $\pi$ donor ligand than the combination of two trans oxo groups, as has been suggested by various researchers. ${ }^{12}$

Cyclic voltammetric studies show that the $\left[\mathrm{ReNL}_{2} \mathrm{Cl}\right]^{+}$ complexes display an irreversible reduction couple at $E_{\mathrm{pc}}$ of $c a$. -2.1 to $-2.4 \mathrm{~V}$ and an irreversible oxidation couple at $E_{\mathrm{pa}}$ of ca. +1.4 to $+1.7 \mathrm{~V}$ vs. ferrocene-ferrocenium in $\mathrm{MeCN}(0.1 \mathrm{~mol}$ $\mathrm{dm}^{-3} \mathrm{NBu}_{4} \mathrm{PF}_{6}$ ) (Table 6). Similarly, irreversible reduction and oxidation couples are observed for the trans $-\left[\mathrm{ReO}_{2} \mathrm{~L}_{2}\right]^{+}$species (Table 6). The irreversible oxidation in both $\left[\mathrm{ReNL}_{2} \mathrm{Cl}\right]^{+}$and trans- $\left[\mathrm{ReO}_{2} \mathrm{~L}_{2}\right]^{+}$is probably ligand-centred in nature. Assuming the reduction process to be metal-centred, a rough estimate of the excited-state reduction potential $E_{\mathrm{f}}\left[\mathrm{ReNL}_{2} \mathrm{Cl}^{+* / 0}\right]$ of $c a$. $0.8 \mathrm{~V}$ es. the normal hydrogen electrode (NHE) can be made for $\mathrm{L}=\mathrm{dppbz}\left[E_{0-0} \approx 2.4 \mathrm{eV}, E_{\mathrm{pc}}=-1.6 \mathrm{~V}\right.$ vs. NHE $]$.

\section{Conclusion}

The nitridorhenium(v) complexes of phosphine and arsine ligands represent a new class of luminescent complexes.* By suitable design of the equatorial ligands and the systematic study of these nitrido and dioxo complexes, a direct comparison of their photophysical as well as spectroscopic properties can be made. insensitive on going from $\mathrm{MeCN}$ to $\mathrm{MeOH}$. This may reflect a

\section{Acknowledgements}

V.W.-W. Y. acknowledges financial support from the University of Hong Kong. K.-K. T. acknowledges the receipt of a postgraduate studentship, administered by the University of Hong Kong.

\section{References}

1 See, for example, J. R. Winkler and H. B. Gray, J. Am. Chem. Soc. 1983, 105, 1373; (b) J. R. Winkler and H. B. Gray, Inorg. Chem., 1985 , 24, 346; (c) H. H. Thorp, J. Van Houten and H. B. Gray, Inorg. Chem., 1989, 28, 889; (d) J. C. Brewer, H. H. Thorp, K. M. Slagle, G. W. Brudvig and H. B. Gray, J. Am. Chem. Soc., 1991, 113,3171; (e) C. M Che, V. W. W. Yam, K. C. Cho and H. B. Gray, J. Chem. Soc., Chem. Commun., 1987, 948; $(f)$ C. M. Che, V. W. W. Yam and W. T. Tang, J. Chem. Soc., Chem. Commun., 1988, 100; $(g)$ V. W. W. Yam and C. M. Che, New J. Chem., 1989, 13, 707; $(h)$ V.W. W. Yam and C. M. Che, Coord. Chem. Rev., 1990, 97, 93; (i) V. W. W. Yam and C. M Che, J. Chem. Soc., Dalton Trans., 1990, 3741; (j) H. Kunkely, T. Türk, C. Teixeira, C de Meric de Bellefon, W. A. Herrmann and A. Vogler, Organometallics, 1991, 10, 2090; $(k)$ C. Reber and J. I. Zink, Inorg. Chem., 1991, 30, 2994.

2 (a) C. D. Cowman, W. C. Trogler, K. R. Mann, C. K. Poon and H. B. Gray, Inorg. Chem., 1976, 15, 1747; (b) M. D. Hopkins, V. M Miskowski and H. B. Gray, J. Am. Chem. Soc., 1986, 108, 6908; (c) C. M. Che, M. H. W. Lam and T. C. W. Mak, J. Chem. Soc., Chem. Commun., 1989, 1529; (d) C. M. Che, T. C. Lau, H. W. Lam and C. K. Poon, J. Chem. Soc., Chem. Commun., 1989, 114.

3 (a) J. Chatt, J. D. Garforth, N. P. Johnson and G. A. Rowe, J. Chem Soc., 1964, 1012; (b) N. P. Johnson, J. Inorg. Nucl. Chem., 1973, 35, 3141; (c) G. F. Ciani, G. D'Alfonso, P. Romiti, A. Sironi and M. Freni, Inorg. Chim. Acta, 1983, 72, 29.

4 R. R. Gagne, C. A. Koval and G. C. Lisensky, Inorg. Chem., 1980, 19, 2854.

5 C. M. Che, K. Y. Wong and F. C. Anson, J. Electroanal Chem. Interfacial Electrochem., 1987, 226, 211.

6 J. C. Brewer and H. B. Gray, Inorg. Chem., 1989, 28, 3334

7 P. W. R. Corfield, R. J. Doedens and J. A. Ibers, Inorg. Chem., 1967, 6 197.

8 K. Yoon, G. Parkin and A. L. Rheingold, J. Am. Chem. Soc., 1991, 113, 1437.

9 R. K. Murmann and E. O. Schlemper, Inorg. Chem., 1971, 10, 2352; C. J. L. Lock and G. Turner, Acta Crystallogr., Sect. B, 1978, 34, 923; P. G. Edwards, A. C. Skapski, A. M. Z. Slawin and G. Wilkinson, Polyhedron, 1984, 3, 1083.

10 J. Chatt, J. D. Garforth, N. P. Johnson and G. Rowe, J. Chem. Soc. A 1969, 2288; N. P. Johnson, J. Chem. Soc. A, 1969, 1843; P. J. Blower and J. R. Dilworth, J. Chem. Soc., Dalton Trans., 1985, 2305.

11 N. P. Johnson, C. J. L. Lock and G. Wilkinson, J. Chem. Soc., 1964, 1054.

12 W. A. Nugent and J. M. Mayer, Metal-Ligand Multiple Bonds, Wiley, New York, 1988

13 G. A. Neyhart, K. J. Seward, J. Boaz and B. P. Sullivan. Inorg. Chem., 1991, 30, 4486; G. A. Neyhart, M. Bakir, J. Boaz, W. J. Vining and B. P. Sullivan, Coord. Chem. Rev., 1991, $111,27$.

* Note added at proof: Photophysical properties of nitridorhenium(v) complexes of diphosphine ligands have recently been reported. ${ }^{13}$ 